\title{
Body mass index as predictor of vasovagal reactions in first time male blood donors
}

\begin{abstract}
Background: Vasovagal reactions (VVR) are common among blood donors and are known to be inversely associated with donor weight. The relationship between VVR and BMI which adjusts donor weight for height is barely explored.
\end{abstract}

Aim: To study the relationship between VVR and BMI.

Method: All whole blood donors in Department of Transfusion medicine, Government Medical College, Thiruvananthapuram over 1year were included in the study. Female and repeat donors were excluded. The weight and height of donors were collected from their donor registration forms. Donors who had reaction during donation and during post donation refreshment period including minor subjective feeling of uneasiness were tested for association with BMI.

Results: BMI was inversely related with VVR, with $11.31 \%$ of donors with $\mathrm{BMI}<18.5$ developing VVR compared to $3.4 \%$ among those who had a BMI $>30$. The mean BMI of donors who had VVR was 22.4 where as donors who did not develop VVR were 22.9.

Conclusion: BMI can be a significant predictor of VVR in donors who fulfill the weight criteria for whole blood donation.

Keywords: body weight, blood phlebotomy, height, blood pressure, blood donors
Volume 2 Issue 5 - 2016

\author{
Abhishekh Basavarajegowda \\ Department of Transfusion medicine, Jawaharlal Institute of \\ Postgraduate Medical Education and Research (JIPMER), India
}

Correspondence: Abhishekh Basavarajegowda, Department of Transfusion medicine, Jawaharlal Institute of Postgraduate Medical Education and Research (JIPMER), Room No I85, Superspeciality Block, Dhanavantrinagar, Pondicherry, India, Tel 9008255888,Email drabhigowda@gmail.com

Received: June 0I, 2016 | Published: August 03, 2016
Abbreviations: VVR, vasovagal reactions; BMI, body mass index; EBV, estimated blood volume

\section{Introduction}

Low body weight among donors is known to be a risk factor for vasovagal reaction during or after whole blood phlebotomy and weight is known to be inversely associated with the reaction rate. ${ }^{1}$ Thin/tall donors are known to have a higher incidence of vasovagal reactions comparatively. The major negative impact of donor reactions are the injury itself, loss of confidence in the injured donors to donate again ${ }^{2}$ and potential discouraging effects on concurrent donors, friends and relatives who are made aware of the event. Many donors who easily weighed more than $50 \mathrm{kgs}$ to qualify as donors still looked very lean due to their discordant height. Vice versa few donors volunteering for donation who looked healthy, stout but short used to be turned off as a result of not weighing $50 \mathrm{kgs}$. This group of people usually completed donation fairly well when the weight was relaxed to $45 \mathrm{kgs}$ in few emergencies and demanding situations (The bag used in our department is for collection of $350 \mathrm{ml}$ of blood). Hence we sought to assess association between having a reaction and BMI which adjusts donor weight for height. BMI provided a simple numeric measure of a person's "fatness" or "thinness". Height and weight of course along with other factors are predictors of estimated blood volume.

\section{Study design and methods}

Design of the study was descriptive. All whole blood donors, donated in department of Transfusion Medicine, at a tertiary care Medical College hospital in south India over 1year from August 2008 up to July 2009 were included in the study. Female and repeat donors were excluded. The weight and height of donors were collected from their donor registration forms. The donors measured their weight by the weighing scale and height by the chart affixed on to the wall in the donor registry room taking help by the or paramedical staff attending them.

\section{Blood collection}

The donor arm with a prominent good ante cubital vein for phlebotomy was located and selected by raising the blood pressure by a BP cuff. After the selection of a suitable vein, the donor arm was prepared by cleaning venipuncture sites at antecubital area starting from center to periphery of the selected arm by betadine and spirit without contamination of selected phlebotomy site, then the phlebotomy was done with minimal trauma and BP set between $40-60 \mathrm{mmHg}$ with continuous pressing of the sponge ball by the same hand to maintain good blood flow. The blood collection was completed within 8-10minutes from the starting time. The blood flow rate was maintained along with continuous mixing. The collected blood units that exceeded total collection time of 10 minutes were excluded from the study as it could be a risk factor for VVR in itself. Donors who had reaction (syncope, vomiting, loss of consciousness and muscular twitches) during donation/post donation/refreshment period including minor subjective feeling of uneasiness were tested for association with BMI.

$$
B M I=\frac{\text { weight in kilograms }}{(\text { height in metres })^{2}}
$$

BMI for a particular height and weight can be calculated by commercially available charts. Statistical analysis were performed by using computer software (SPSS version 10.0) 


\section{Results}

A total of 9569 male first time donors were included in the study. Among the participants $12 \%$ were having less than 18.5 BMI, 33\% had a BMI ranging from 18.5 to $22,29 \%$ belong to $22-25$ BMI group, $21 \%$ were in 25 to $30 \mathrm{BMI}$ and $5 \%$ had a BMI $>30$. The Distribution of donors in relation to BMI status is depicted in Figure 1. The comparison of BMI in relation to the VVR is given in Table 1. The mean BMI among the donors with no VVR reactions was $22.9( \pm 3.7)$ and among the donors with VVR, it was 22.4( \pm 3.1$)$. It shows that the mean BMI was significantly lower $(\mathrm{P}<0.05)$ in donor who had VVR compared to the donors with no VVR.

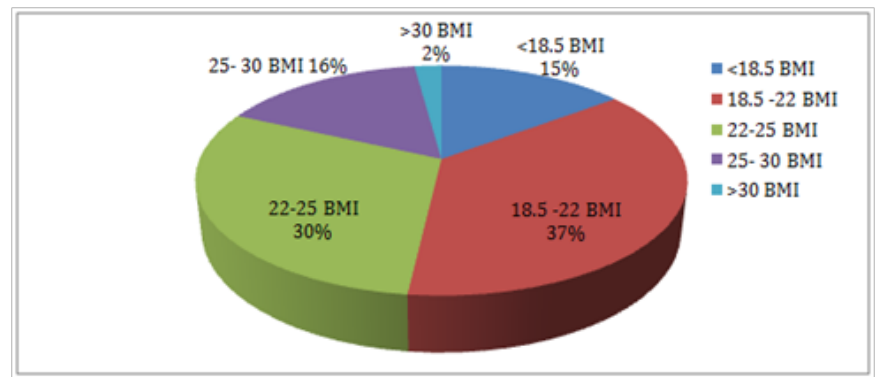

Figure I Distribution of donors in relation to BMI status.

Table I Comparison of BMI in relation to BMI

\begin{tabular}{lllll}
\hline Group & Number & Mean & SD & P-Value \\
\hline VVR & 849 & 22.4 & 3.1 & \\
NoVVR & 8720 & 22.9 & 3.7 &
\end{tabular}

The incidence of VVR in different BMI groups is depicted in Figure 2. The incidence of VVR among the donors with $<18.5$ BMI was $11.3 \%$ followed by $10 \%$ in donors with BMI $18.5-22$. The incidence if VVR decreases with increasing BMI as shown in the Figure 2 and the trend in the decrease of the incidence of VVR in relation to the BMI status was found to be statistically significant $(\mathrm{P}<0.05)$.

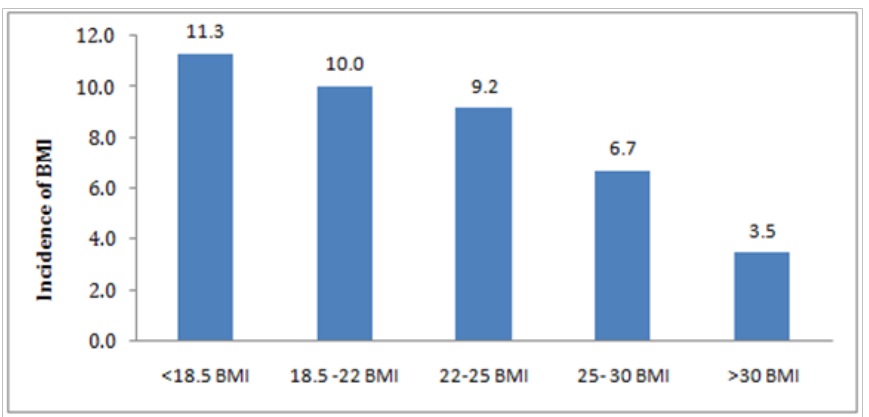

Figure 2 Incidence of VVR reactions in different BMI groups.

\section{Discussion}

The various factors that predict an adverse reaction in whole blood donors are young age, female sex, first time donors, non black, ${ }^{3}$ low BMI ${ }^{4}$ small estimated blood volume, rapid pulse and low systolic/ diastolic blood pressure. ${ }^{5}$ The greatest predictor of a reaction has been argued to be low blood volume followed by young age.

Total blood volume is directly proportional, although not perfectly to body weight. Other factors like body height and relative muscle mass also affect total blood volume. Nadler et al. ${ }^{6}$ devised a formula to calculate blood volume from height and weight differently for both sexes. ${ }^{6}$ It is recognized that at least 5 percent of blood donors selected by weight of more than $50 \mathrm{~kg}$ had blood volumes of less than $3500 \mathrm{ml} .{ }^{6}$ The formula given by Nadler et al. ${ }^{6}$ is cumbersome to use and needs either software or a designated person for its calculation and time consuming to be used at donor registry. It's suggested that Nadler's formula overestimates blood volume by as much as $12 \%$. BMI was explicitly cited by Ancel Keys as being appropriate for population studies. Due to its simplicity, it came to be widely used for individual diagnosis, despite its inappropriateness. For a given height and weight BMI values can be obtained by looking into the charts which are commercially available. A donor who has a weight of $55 \mathrm{~kg}$ who would be suitable as a donor by weight alone has only a BMI of around 17 due to their sheer height thereby putting them at risk of VVR whereas donors with just around 50 with height of $160 \mathrm{~cm}$ would cope well with donation as their BMI is well in 23 to 25 .

BMI values are age independent and the same for both the sexes. ${ }^{8}$ When weight alone is used as a criterion for blood donation, many donors who fulfill it, may still be at a high risk for VVR due to their low BMI. So identifying this group and giving extra attention during and after donation may reduce the chances of reaction. Rios et al. ${ }^{7}$ in a huge study comprising 5,91 and 177 donors concluded that deferral of donors with low Estimated Blood Volume (EBV) may offer a rational approach in protecting donors at greatest risk from vasovagal reactions without jeopardizing the blood supply to a greater extent. Our study shows a gradual decrease in VVR rates as the BMI increases linearly as been shown by Narbey et al. ${ }^{9}$ in their study that higher BMI is protective for both men and women against vasovagal reactions. ${ }^{9}$ Our study was done in only males and first time donors to avoid gender and donation status as confounding factors. However age still remains as an important factor which predisposes to VVR as younger age has a higher risk which we have not taken into account although all of our donors were within 50years of age. Since ours was a single centre study from donors usually coming from the surrounding areas we expect them to homogenous in respect ethnicity with few minor exceptions. We did not try to analyze other factors like pulse, blood pressure etc. Directing donors with low BMI to singleunit red cell apheresis with saline replacement may reduce the risk of reactions. ${ }^{10}$ Selection of alternative collection volumes (reduced volume donation) would also feasible depending on the policies of the centre and needs of the hospitals they cater to. Likewise donors who were otherwise deferred due to low weight alone would do well after donation due to their adequate BMI. Our study could not show that BMI is an independent predictor of VVR. Further studies may be required to find out a definite BMI above which VVR may be reduced.

\section{Acknowledgements}

None.

\section{Conflict of interest}

The author declares no conflict of interest.

\section{References}

1. Newman BH. Vasovagal reaction rates and body weight. Transfusion. 2003;43(8):1084-1088

2. Newman BH, Newman DT, Ahmad R, et al. The effects of whole blood donor adverse events on blood donor return rates. Transfusion. 2006;46(8):1374-1379.

3. Newman BH. Vasovagal reactions in high school students: findings relative to race, risk factor synergism, female sex and non high school participants. Transfusion. 2002;42(12):1557-1560. 
4. Rios JA, Fang JY, Spencer BR, et al. Relationship between BMI and other factors in blood donors having vasovagal reactions. Transfusion. 2008;48:30A.

5. Wiltbank TB, Giordano GF, Kamel H, et al. Faint and prefaint reactions in whole blood donors. Transfusion. 2008;48(9):1799-1808.

6. Nadler SB, Hidalgo JH, Bloch T. Prediction of blood volume in normal human adults. Surgery. 1962;51(2):224-232.

7. Rios JA, Fang J, Tu Y, et al. The potential impact of selective donor deferrals based on estimated blood volume on vasovagal reactions and donor deferral rates. Transfusion. 2010;50(6):1265-1275.
8. Park K. Park's textbook of preventive and social medicine. 19th ed. Brazil; 2007. 334 p.

9. Narbey D, Fillet AM, Jbilou S, et al. Case-control study of immediate and delayed vasovagal reactions in blood donors. Vox Sang. 111(3):257-265.

10. Trend JJT, Cable RG, Badon SJ, et al. A case-controlled multicenter study of vasovagal reactions in blood donors: influence of sex, age, donation status, weight, blood pressure, and pulse. Transfusion. 1999;39(3):316320 . 